\title{
A long-term Survival in a Patient with Metastatic DDR2 Positive Adrenal Cortical Carcinoma
}

\author{
Taza $\mathrm{F}^{1^{*}}$, Chovanec $\mathrm{M}^{1,2}$, Noah Hahn ${ }^{3}$, Albany $\mathrm{C}^{1}$ \\ ${ }^{1}$ Division of Hematology and Oncology, Indiana University Simon Cancer Center, \\ Indianapolis, IN, USA \\ ${ }^{2} 2^{\text {nd }}$ Department of Oncology, Faculty of Medicine, Comenius University and National \\ Cancer Institute, Bratislava, Slovakia \\ ${ }^{3}$ The Johns Hopkins University School of Medicine \\ *correspondence to Fadi Taza \\ Email: ftaza@iu.edu
}

Clinical Practice Points:

- Adrenal cortical carcinoma (ACC) is a rare malignancy with poor prognosis.

- The high rate of recurrence in this disease emphasizes the necessity for careful clinical and radiographic follow-up for timely diagnosis of a recurrence.

- Patients with recurrent or metastatic disease should undergo repeat surgery, if the tumor is potentially resectable, because surgery may significantly prolong median survival.

- In this brief report, a 57-year old male patient with recurrent and metastatic ACC achieved long term survival of 11 years with surgery, chemotherapy and mitotane.

- Whole genome sequencing of tumors can shed light on possible therapeutically relevant events that might help inform treatment recommendations for advanced cancers. 
Keywords: Adrenal cortical carcinoma (ACC), mitotane, long term survival, genome sequencing, DDR2

\section{Introduction}

Adrenocortical carcinoma (ACC) is a rare malignancy with an estimated worldwide annual incidence of one to two per million [1]. It is responsible for only approximately $0.2 \%$ of all cancer deaths in the United States annually, and it is the second most aggressive endocrine malignancy following anaplastic thyroid cancer [2]. ACC carries a poor prognosis with an expected 5-year survival of $13 \%$ in patients stage IV disease [3]. Surgery is the mainstay treatment in localized ACC and creates the best hope for prolonged survival and potential cure. However, $60 \%$ of patients who present with localized disease experience recurrence that often requires systemic therapy [4].

Herein, we present a case report of a recurrent and metastatic ACC with a rare discoid domain receptor-2 (DDR2) mutation with an ongoing long-term survival more than 11 years achieved with surgery, chemotherapy and mitotane retreatments. To date, only eight case reports, one small case series of six patients and 12 patients from multicenter retrospective 
analysis in the literature have reported on patients with ACC who have achieved long-term survival of more than 10 years [5-14]. The 5-year and 10 -year survival of patients with metastatic disease reported by Tran et al from the multicenter retrospective analysis was $27 \%$ and $7 \%$, respectively [14].

\section{Case report}

A 57-year old man presented with left flank pain on February 2006. Computed tomography (CT) scan of the abdomen revealed a $4.5 \mathrm{~cm}$ left adrenal mass. He underwent a fine needle biopsy with a cytological finding suggestive of a malignant tumor. A complete metabolic evaluation for pheochromocytoma was negative. Subsequently, he elected for laparoscopic left adrenalectomy. Histological finding confirmed an adrenal cortical carcinoma with negative margins, no capsule involvement, low to moderate mitotic activity and the presence of vascular invasion. Postsurgical CT staging examination showed no evidence of metastatic disease and the diagnosis of a low risk stage I ACC was made. The patient was enrolled into a routine follow-up with regular CT scans, clinical and laboratory examinations for the first 2 years postoperatively. He was free of recurrence during these visits, and then he was lost to follow up for 4 years until November 2012 when he presented with symptoms of gross hematuria and back pain.

Radiologic studies at that time revealed a recurrent disease with left adrenal mass abutting the left kidney and spleen, left retroperitoneal mass and left para spinal soft-tissue mass (Figure 1A). In January 2013, He underwent a retroperitoneal lymph node dissection and left partial nephrectomy with pathological diagnosis of $5.2 \mathrm{~cm}$ recurrent left adrenal cortical carcinoma invading left kidney and spleen with positive pancreatic margins. He remained disease free until February 2014 when the CT evaluation uncovered several liver lesions (Figure 1B). A liver biopsy confirmed metastatic adrenal cortical carcinoma. Subsequently, the patient was treated with six cycles of etoposide, doxorubicin and carboplatin (EDP), with concomitant oral mitotane (the dose titrated according to serum mitotane levels)[15], until July 2014 when the CT scan revealed no evidence of progression or new metastases. He progressed with numerous bilateral small pulmonary nodules on CT images in January 2015, but the liver remained disease-free (Figure 
1C). As a result, he was reintroduced to a daily single agent treatment with mitotane, which he continues to take until present time.

Whole genome sequencing from the relapsed resected tumor mass, was performed. The tumor was largely devoid of mutations, copy number alterations or translocations. However, a discoid domain receptor-2 (DDR2) missense mutation was discovered. The tumor sample's exonic mutation rate was estimated to be 0.5 mutations per megabase.

The patient is still alive with radiology studies showing stable pulmonary nodules and no evidence of progression on 2.5 grams of daily mitotane alone. His long-term survival has reached more than 11 years at the last follow up in April 2017.

\section{Discussion}

ACC is a rare endocrine malignancy. A complete resection of the primary tumor is the only potentially curative treatment. However, even in patients who undergo radical adrenalectomy for a stage I disease, the recurrence is common [4]. A prognosis of a metastatic disease is dismal. The treatment of recurrence has been predominantly based on chemotherapy and mitotane, although some patients may benefit from surgical resection of a disease that is anatomically confined. The overall survival of EDP plus Mitotane is about 14 months [15]. Mitotane is currently a cornerstone in the management of cortisol secreting ACC inducing adrenal cortex necrosis, mitochondrial membrane impairment, and irreversible binding to CYP proteins including CYP11A1, CYP11B1, CYP11B2, and 3b-hydroxysteroid-dehydrogenase $[16,17]$. However, the degree of response to mitotane is variable among patients due to the complexity of its mechanisms of action, pharmacokinetics and tolerability. The course of this patient was remarkable because he achieved an ongoing long-term survival with metastatic ACC after treatment with mitotane and chemotherapy. The finding of the genome largely devoid of mutations with the exception of single discoid domain receptor-2 (DDR2) mutation in this patient could be a contributing factor to his excellent survival and high sensitivity to mitotane. In contrast to our patient, ACC is generally known to have a high mutational burden $[18,19]$.

DDR2 is a receptor tyrosine kinase that interacts with collagen and plays a role in cell adhesion 
and proliferation [20]. DDR2 was found to be mutated in approximately $4 \%$ of patients with lung squamous cell carcinoma [21], lung adenocarcinoma, gastric and breast cancer but not in ACC $[18,19]$. Recent report from pre-clinical cell line and tissue-based model suggests that somatic mutations in the (DDR2) are responsive to dasatinib (a DDR2 receptor tyrosine kinase inhibitor) [22]. Early case reports have also reported sensitivity to dasatinib in squamous cell carcinoma of the lung [23]. Based on the results from the patient tumor whole genome sequencing we hypothesize, that treatment with dasatinib could be a meaningful approach in case of further progression of the disease.

Whole genome sequencing can identify tumor drivers and potential actionable mutations, generally brings detailed information on tumor heterogeneity and guides clinicians in decisions making for treatment recommendations in advanced cancers. We suggest whole genome sequencing in selected patients who have previously failed standard treatments and/or in patients with unexpected biological behavior of the ACC.

\section{Conclusion}

This case report demonstrates an exceptionally rare long-term survival of a patient with metastatic ACC. His survival exceeding 11 years was achieved by chemotherapy, surgery and mitotane retreatments. A rare DDR-2 mutation was discovered, that could be illustrative of a different biological behavior of ACC in our patient.

\section{Acknowledgements}

This work was supported by the Slovak Research and Development Agency under contract No. APVV-0016-11 and APVV-15-0086 for Michal Chovanec.

\section{Disclosure}

Authors declare no conflict of interest 


\section{References}

1. Allolio, B. and M. Fassnacht, Clinical review: Adrenocortical carcinoma: clinical update. J Clin Endocrinol Metab, 2006. 91(6): p. 2027-37.

2. Schteingart, D.E., et al., Management of patients with adrenal cancer: recommendations of an international consensus conference. Endocr Relat Cancer, 2005. 12(3): p. 667-80.

3. Fassnacht, M., et al., Limited prognostic value of the 2004 International Union Against Cancer staging classification for adrenocortical carcinoma: proposal for a Revised TNM Classification. Cancer, 2009. 115(2): p. 243-50.

4. Ayala-Ramirez, M., et al., Adrenocortical carcinoma: clinical outcomes and prognosis of 330 patients at a tertiary care center. Eur J Endocrinol, 2013. 169(6): p. 891-9.

5. Bergeat, D., et al., An unusual case of adrenocortical carcinoma with liver metastasis that occurred at 23 years after surgery. Hepatobiliary Surg Nutr, 2016. 5(3): p. 265-8.

6. De Leon, D.D., et al., Long-term (15 years) outcome in an infant with metastatic adrenocortical carcinoma. J Clin Endocrinol Metab, 2002. 87(10): p. 4452-6.

7. Hermsen, I.G., et al., Extremely long survival in six patients despite recurrent and metastatic adrenal carcinoma. Eur J Endocrinol, 2008. 158(6): p. 911-9.

8. Ilias, I., et al., Sustained remission of metastatic adrenal carcinoma during long-term administration of low-dose mitotane. J Endocrinol Invest, 2001. 24(7): p. 532-5.

9. Mawardi, M., B. Al-Judaibi, and P. Marotta, Hepatic metastasis from adrenocortical carcinoma fifteen years after primary resection. Saudi J Gastroenterol, 2012. 18(2): p. $140-2$.

10. Meyer, A. and M. Behrend, 32-year survival with metastatic adrenal cortical carcinoma-update of a case report. Anticancer Res, 2007. 27(2): p. 1045-6.

11. Orlando, R., M.R. Pelizzo, and F. Lirussi, Adrenocortical carcinoma: a 15-year survival after complete resection and repeated resection. A retrospective study in a patient with an expected poor prognosis. Anticancer Res, 2003. 23(3C): p. 2929-31. 
12. Sakamoto, K., A. Ariyoshi, and M. Okazaki, Metastatic adrenocortical carcinoma treated by repeated resection: a case report of long-term survival over 18 years. Int J Urol, 1995. 2(1): p. 50-2.

13. van Aalderen, W., et al., A case of recurrent adrenocortical carcinoma, with observations on long-term o, $p^{\prime}-D D D$ therapy and complications. Neth J Med, 1992. 41(3-4): p. 161-70.

14. Tran, T.B., et al., Actual 10-year survivors following resection of adrenocortical carcinoma. J Surg Oncol, 2016. 114(8): p. 971-976.

15. Fassnacht, M., et al., Combination chemotherapy in advanced adrenocortical carcinoma. N Engl J Med, 2012. 366(23): p. 2189-97.

16. Lin, C.W., Y.H. Chang, and H.F. Pu, Mitotane exhibits dual effects on steroidogenic enzymes gene transcription under basal and cAMP-stimulating microenvironments in NCI-H295 cells. Toxicology, 2012. 298(1-3): p. 14-23.

17. Fassnacht, M., M. Kroiss, and B. Allolio, Update in adrenocortical carcinoma. J Clin Endocrinol Metab, 2013. 98(12): p. 4551-64.

18. Gao, J., et al., Integrative analysis of complex cancer genomics and clinical profiles using the cBioPortal. Sci Signal, 2013. 6(269): p. pl1.

19. Cerami, E., et al., The cBio cancer genomics portal: an open platform for exploring multidimensional cancer genomics data. Cancer Discov, 2012. 2(5): p. 401-4.

20. Leitinger, B., Discoidin domain receptor functions in physiological and pathological conditions. Int Rev Cell Mol Biol, 2014. 310: p. 39-87.

21. Hammerman, P.S., et al., Mutations in the DDR2 kinase gene identify a novel therapeutic target in squamous cell lung cancer. Cancer Discov, 2011. 1(1): p. 78-89.

22. Day, E., et al., Inhibition of collagen-induced discoidin domain receptor 1 and 2 activation by imatinib, nilotinib and dasatinib. Eur J Pharmacol, 2008. 599(1-3): p. 44-53.

23. Pitini, V., et al., Response to dasatinib in a patient with SQCC of the lung harboring a discoid-receptor-2 and synchronous chronic myelogenous leukemia. Lung Cancer, 2013. 82(1): p. 171-2. 
Figure 1. Treatment outcome in a patient with metastatic ACC treated with surgery, chemotherapy and mitotane.

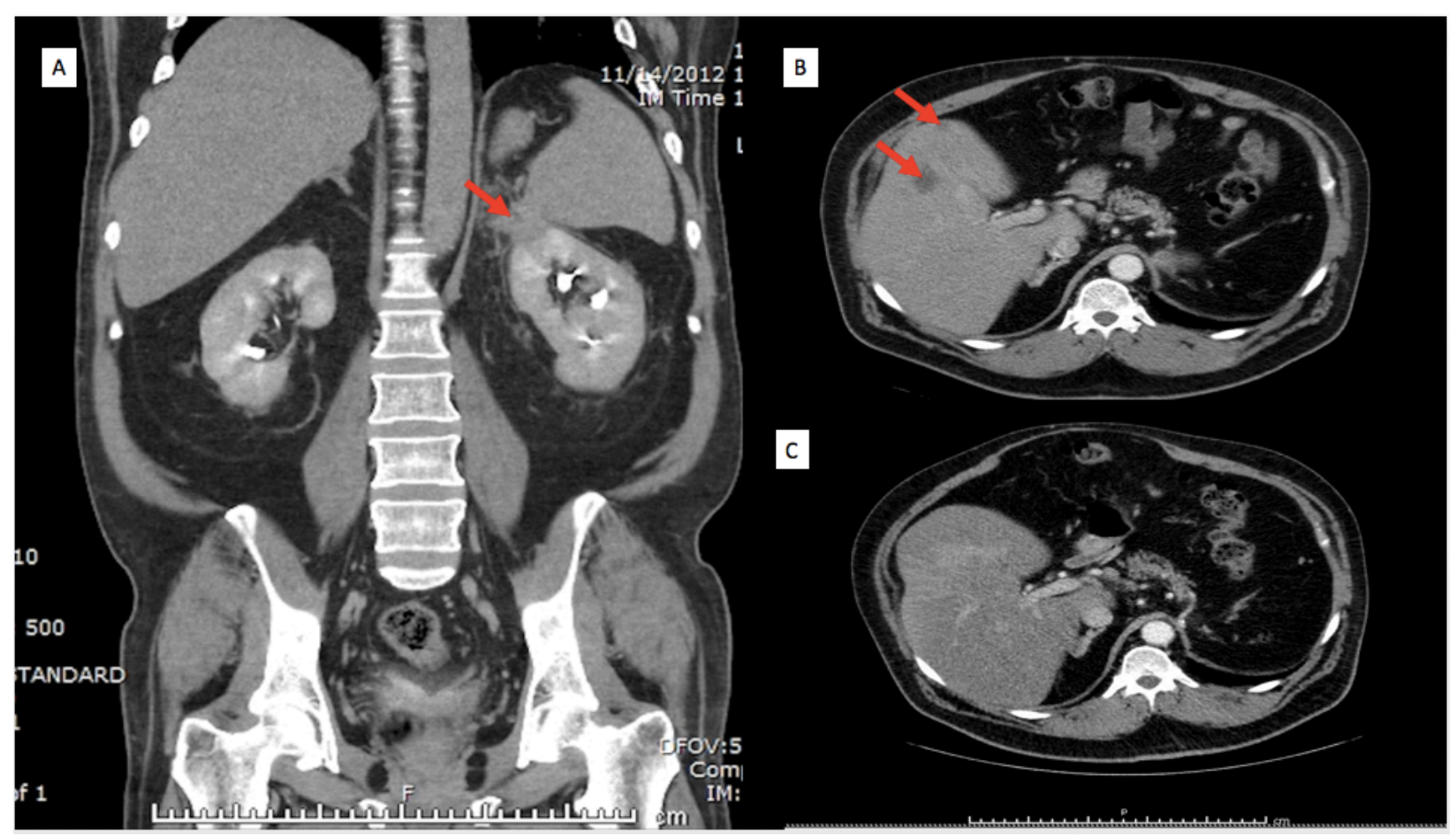

Figure legend: (A) left adrenal mass abutting the left kidney and spleen before surgery. (B) liver metastases before chemotherapy and mitotane treatment. (C) No evidence of Liver metastases on follow-up Computed tomography scan. 